\title{
An uncommon recurrence of an immature teratoma: A case report
}

\author{
XUELU LI ${ }^{1 *}$, DANDAN ZHU ${ }^{2 *}, \mathrm{LI} \mathrm{LV}^{3}$ and JIAN YU ${ }^{2}$ \\ Departments of ${ }^{1}$ Breast Surgery, ${ }^{2}$ Critical Care Medicine and ${ }^{3}$ Pathology, \\ The Second Hospital of Dalian Medical University, Dalian, Liaoning 116023, P.R. China
}

Received March 1, 2015; Accepted November 19, 2015

DOI: $10.3892 / 01.2016 .4254$

\begin{abstract}
A 24-year-old female who was previously diagnosed with an immature, right ovarian teratoma was referred to The Second Hospital of Dalian Medical University (Dalian, China) due to abdominal pain. The patient had previously undergone a laparotomy and right ovarian oophorectomy. Despite this, at 10 years post-surgery, the patient developed extensive abdominal metastases, in conjunction with elevated serum cancer antigen (CA)-125 and CA19-9 levels. The patient had a high risk of recurrence, and the liver and spleen metastases were subsequently diagnosed as mature teratomas. In order to prevent the possibility of disease recurrence, frequent post-surgical follow-up, consisting of imaging analysis and the testing of serum markers, is required. Regarding the published literature, there have been no other patients presenting with a giant teratoma with associated metastasis. Due to the lack of regular follow-up appointments in China, the tumor in the present study was not recognized until too late. Therefore, to increase the successful detection of recurrent teratomas, a longer follow-up period is necessary for patients who present with a high risk of recurrence.
\end{abstract}

\section{Introduction}

Teratomas may be categorized as either mature or immature, and originate from the three pluripotent germ cell layers: The ectoderm (skin and neural tissue), the endoderm (gastrointestinal and bronchial epithelium, and thyroid tissue), and the mesoderm (fat, bone, muscle and cartilage) (1). Immature teratomas are rare tumors that develop in the ovaries, accounting for $0.25-3 \%$ of tumors that develop in the ovaries, with individuals at a greater risk during their first two decades of life (1). By contrast, mature teratomas occur most commonly in women aged between

Correspondence to: Dr Jian Yu, Department of Critical Care Medicine, The Second Hospital of Dalian Medical University, 467 Zhong Shan Road, Dalian, Liaoning 116023, P.R. China E-mail:dmulixl@163.com

*Contributed equally

Key words: teratoma, recurrence, metastasis, growing teratoma syndrome
20-40 years (2). Teratomas have high recurrence and metastasis rates, and immature tumor tissues may be converted into mature tissues following post-surgical recurrence. The conversion of immature teratoma is characterized by slow growth, so symptoms are not typical. Clinical doctors often neglect the diagnosis of teratomas. If the size of the tumor is $>6 \mathrm{~cm}$, it is common for surgeons to utilize a surgical treatment. Recurrence typically develops subsequent to surgical excision of the tumor, often within the first year of primary therapy (3). The present study describes the case of a patient who underwent excision of an immature teratoma, which later developed into mature teratomas found as extensive abdominal metastases to the liver, spleen and pelvic cavity, identified after 10 years.

\section{Case report}

A 24-year-old female was referred to the Department of General Surgery, The Second Hospital of Dalian Medical University (Dalian, China), on 24 April 2013, due to intermittent upper abdominal pain. Upon physical examination of the right abdomen, a hard, large mass that moved under palpation was identified. Abdominal tenderness, rebound pain and muscular tension were impalpable, with no other positive signs. When examining the medical history of the patient in detail, an immature right ovarian teratoma that had been diagnosed 10 years previously was noted, for which the patient had undergone a laparotomy and right ovarian oophorectomy. The post-surgical pathological analysis had identified an immature teratoma, with an official diagnosis of grade 2 immature teratoma [Norris grading system (4)]. Adjuvant therapy had been administered, consisting of 3 cycles of bleomycin $\left(20 \mathrm{U} / \mathrm{m}^{2}\right)$, etoposide $\left(1,675 \mathrm{mg} / \mathrm{m}^{2}\right)$ and cisplatin $\left(20 \mathrm{mg} / \mathrm{m}^{2}\right)$, following surgery. At the last chemotherapy session, no residual tumor had been observed. The patient had subsequently been lost to follow-up.

Based on the medical history of the patient, recurrence of the teratoma was now suspected. Notably, laboratory tests exhibited elevated levels of the serum markers cancer antigen (CA)-125 and CA19-9 (Table I). In order to assess the giant tumor in the abdomen, the patient underwent a sonographic examination and computed tomography (CT) scan. The contrast-enhanced total abdominal CT scan revealed a complex cystic mass, measuring $18.6 \times 15.7 \mathrm{~cm}^{2}$, on the right lobe of the liver (Fig. 1A). Additionally, a further tumor that exhibited the same properties as the giant mass was detected on the spleen (Fig. 1B). 
Table I. Dynamic change of tumor markers.

\begin{tabular}{lccccc}
\hline & & \multicolumn{3}{c}{ Level post-surgery, kU/1 } & Normal \\
\cline { 3 - 5 } Tumor marker & $\begin{array}{c}\text { Level prior } \\
\text { to surgery, kU/1 }\end{array}$ & 7 days & 30 days & 180 days & range, kU/1 \\
\hline CA19-9 & $>7000.00$ & 1927.51 & 250.50 & 23.00 & $0.00-35.00$ \\
CA12-5 & 440.63 & 201.70 & 40.00 & 5.20 & $0.00-35.00$ \\
\hline
\end{tabular}

CA, cancer antigen.
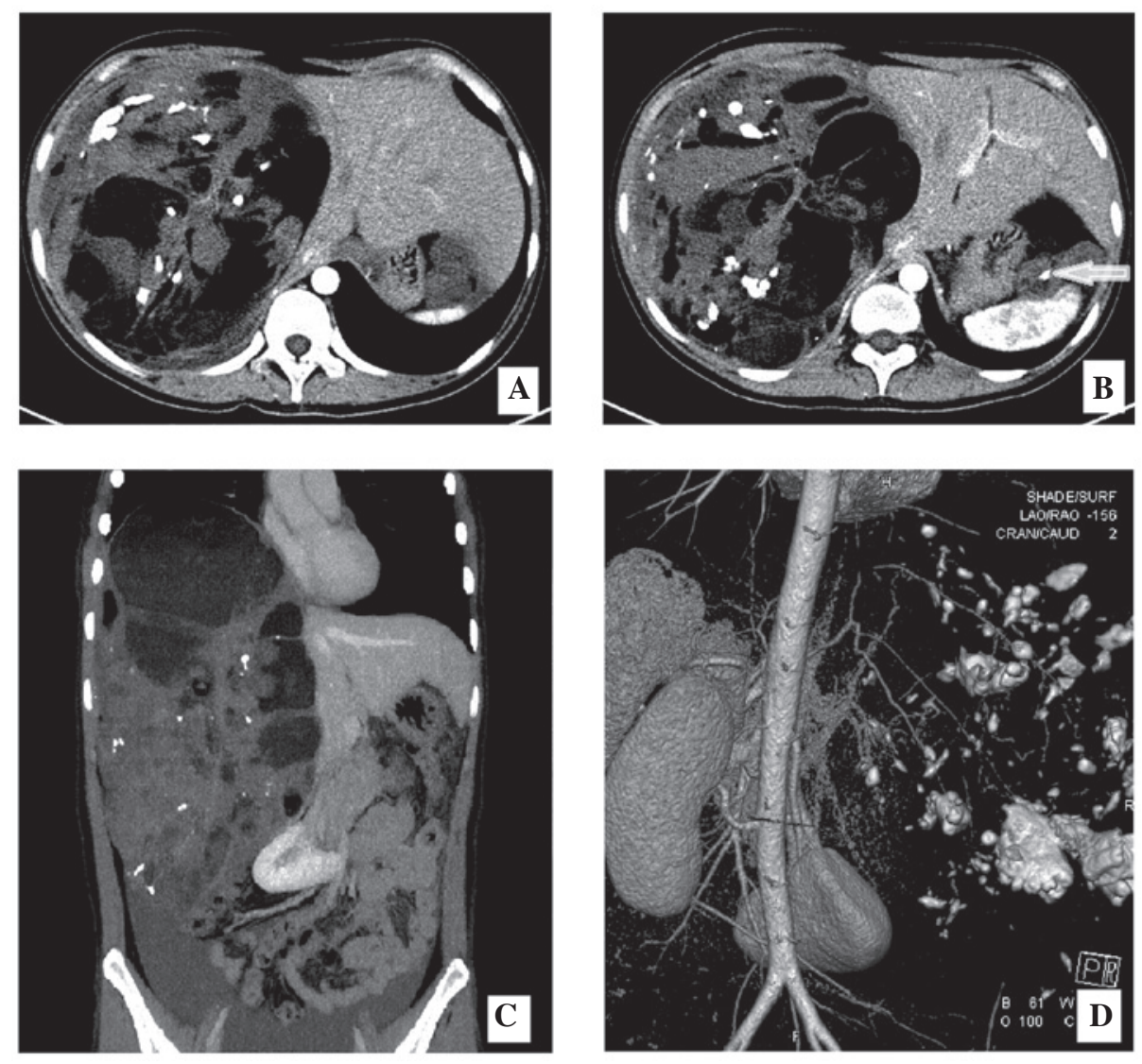

Figure 1. (A) Abdominal computed tomography enhancement scanning. A mass $\left(18.6 \times 15.7 \mathrm{~cm}^{2}\right)$ was identified on the right liver lobe, exhibiting calcification and cystic changes. (B) A mass was also identified on the surface of the spleen, exhibiting calcification (white arrow). (C) An abdominal coronal section demonstrated that the compressed right ventricle, right kidney and pancreas had shifted to the left, and ascites was observed in the pelvic cavity. (D) Vascular remodeling.

With consideration of the tumor size, the compression symptoms (the right ventricle, right kidney and pancreas had shifted to the left; Fig. 1C and D) and the elevated serum markers, a diagnosis of an immature teratoma was the most probable. The patient subsequently underwent a laparotomy. Possible invasion of the tumor into the liver could not be excluded, therefore, a cardiopulmonary blood bypass was prepared. Following entry into the peritoneal cavity, the mass was removed from the right lobe of the liver, the first porta hepatis was blocked and the right hepatic vein was resected where the tumor had infiltrated. Subsequently, complete removal of the mass from the spleen surface was successful. The giant tumor was rich in sebaceous materials, with hair shaft tissues and teeth identified inside the mass (Fig. 2A and B). The pathology report confirmed that all tumors on the surface of the liver and the spleen, and in the pelvic cavity were mature teratomas (Fig. 3). Following tumor resection, there was no evidence of recurrence or metastasis during the 1 and 6 month follow-up evaluations and ongoing follow-up examinations every 3-6 months have been planned.

\section{Discussion}

Immature teratomas of the ovary are rare tumors, accounting for $<3 \%$ of all teratomas located in the ovaries. The disease typically occurs during the first two decades of life, and the tumors are composed of tissue derived two or more germ cell layers (ectoderm, mesoderm and/or endoderm), containing immature and embryonal structures (1). The current case presented with the classic symptoms and histological appearance associated with immature teratomas. Excision of such tumors is often followed by local recurrence within the first year of primary 

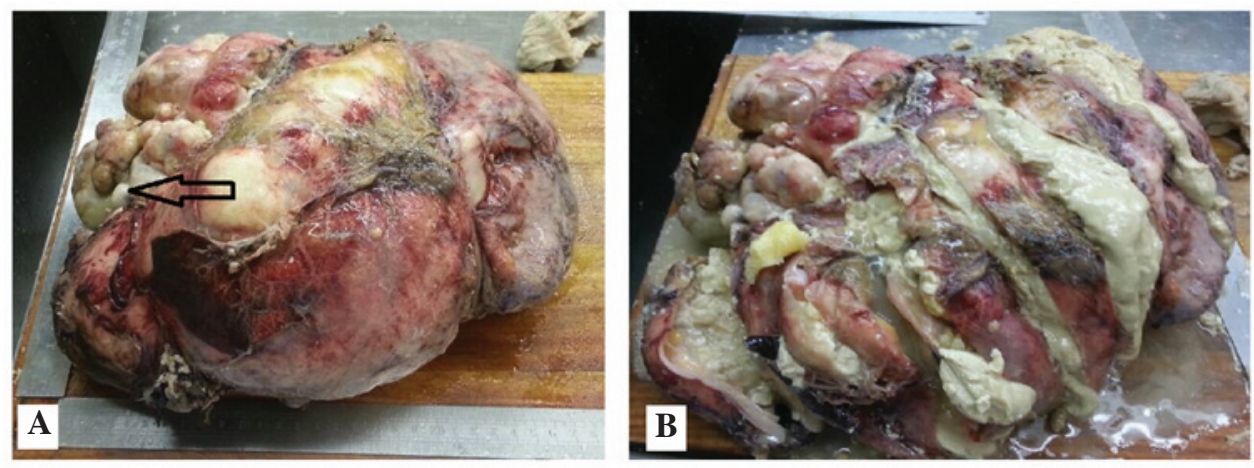

Figure 2. Gross specimen removed from the liver. (A) The tumor $\left(29 \times 24 \mathrm{~cm}^{2}\right)$ contained hair shaft tissues and teeth (black arrow). (B) The inside of the mass contained abundant, gelatinous and sebaceous materials, hair shaft tissues and teeth.
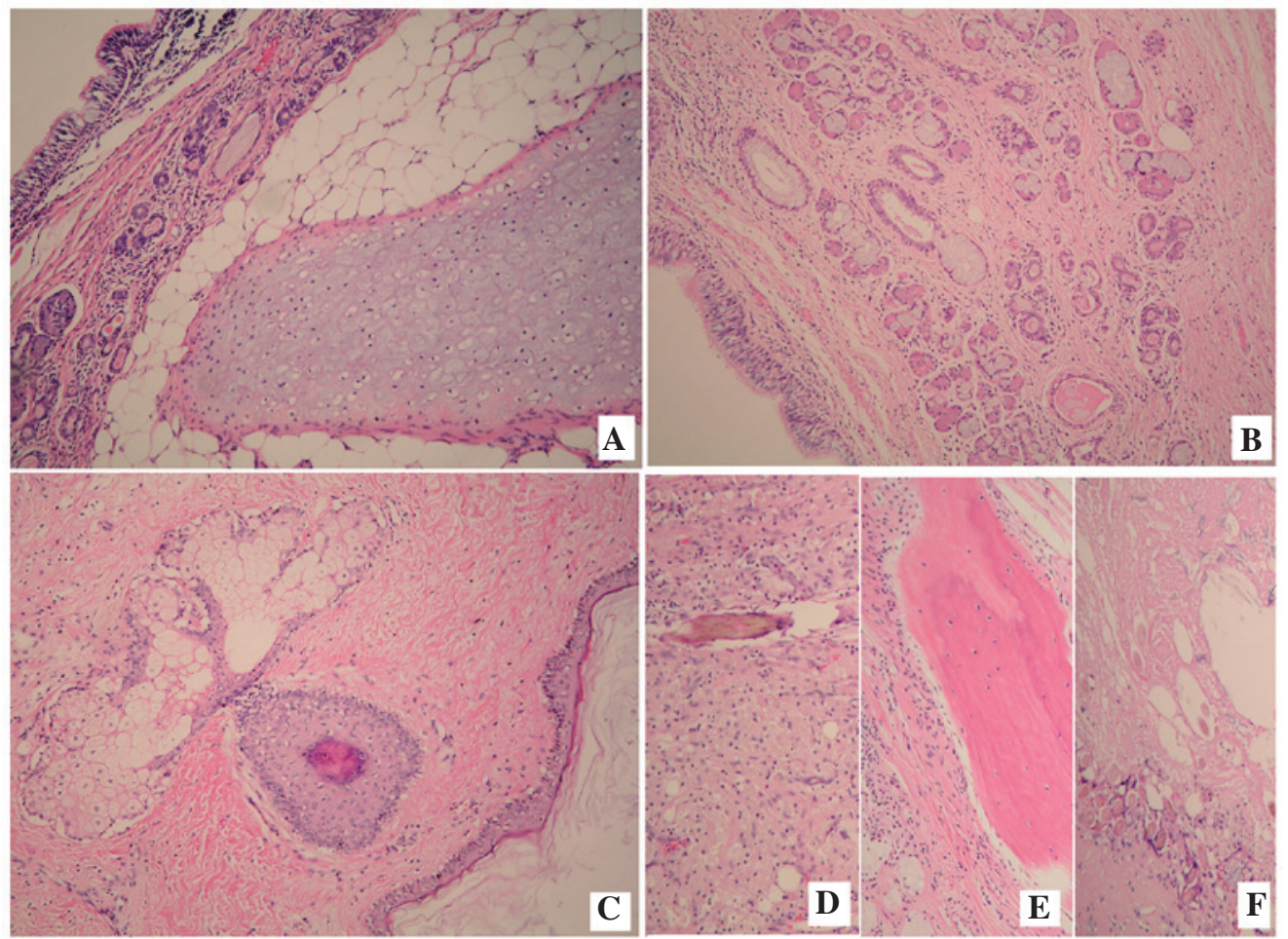

Figure 3. Hematoxylin and eosin staining features. (A) Mature components of the bronchial epithelial cells, the bronchial glands and the bronchial cartilage. (B) Typical bronchial epithelium. Photomicrographs showing the skin tissue with (C) sebaceous glands, and (D) hair, (E) lamellar bone and (F) calcification. Magnification, $x 100$.

therapy initiation (3). The present patient was lost to follow-up subsequent to initial chemotherapy, but developed rare mature teratomas and extensive abdominal metastases after 10 years.

Mature teratomas are typically benign, however, malignant transformation may occur, albeit rarely. The peak incidence of such tumors is reported in women between the ages of 20-40 years. The disease is characterized by slow growth, with a growth rate of $1.8 \mathrm{~mm}$ per year, and when the size of the tumor is $<6 \mathrm{~cm}$, it is common for surgeons to utilize a non-surgical treatment (1). Growing teratoma syndrome is an uncommon condition present in men and women, with appropriately treated germ cell tumors characterized by the persistence or development of enlarging masses following adjuvant chemotherapy, normal tumor marker values and the presence of mature teratomas in the specimen. If a diagnosis of growing teratoma syndrome is confirmed, the surgeon must determine the operability of the tumor, whilst also weighing up the risks and benefits of surgery (5). This is necessary to allow for the optimal management of the disease and to prevent unnecessary chemotherapy. In the present case, the serum makers, CA-125 and CA19-9, exhibited particularly high levels when compared with levels observed in growing teratoma syndrome. Excision was performed on tumors that were $<2 \mathrm{~cm}$ in size. Following the surgery, pathological sections from different locations in the tumor showed no immature elements. No further treatment was administered to the patient. To the best of our knowledge, this metastasis of a mature teratoma to the liver, the spleen and the pelvic cavity is particularly rare.

When forming a differential diagnosis for teratomas, the measurement of the serum levels of CA-125 and CA19-9 is a 
common method used for early detection. Ustunyurt et al (6) reported that serum CA19-9 is the most reliable tumor marker of mature ovarian cystic teratomas, and that the levels of serum CA19-9 are often associated with the size of the teratoma; however, this does not appear to have high specificity or sensitivity. Bast et al (7) reported that serum CA-125 is a reliable marker, and that in combination with elevated CA19-9, it may be a differential character of malignant tumors. Notably, in the present case, the variation in serum CA-125 and CA19-9 levels was greater compared with the typical change (Table I).

Harada et al (8) hypothesized that a young age ( $<30$ years), a large cyst size $(>8 \mathrm{~cm})$ and the bilateral occurrence of mature teratomas are predictive factors for recurrence. In the present case, the patient was at a high risk of recurrence. In order to prevent residual disease, frequent post-surgical imaging analysis and the testing of serum markers is required. In the literature, to the best of our knowledge, no giant teratoma patients have been described who have presented with the same uncommon metastasis as the patient in the current case. Due to the lack of follow-up appointments in China, the tumor was recognized too late. To increase the likelihood of detecting recurrent teratomas, longer follow-up periods are required for patients who are at a high risk of recurrence.

\section{References}

1. Saba L, Guerriero S, Sulcis R, Virgilio B, Melis G and Mallarini G: Mature and immature ovarian teratomas: CT, US and MR imaging characteristics. Eur J Radiol 72: 454-463, 2009.

2. Chang CF and Lin CK: A case of recurrent, bilateral ovarian mature teratoma in a young woman. BMC Womens Health 14 : 57, 2014.

3. Barwad A, Dey P and Shivalingam J: Metastatic of mature component in a treated case of immature teratoma diagnosed on fine-needle aspiration cytology of the liver. Diagn Cytopathol 39: 711-713, 2011.

4. Norris HJ, Zirkin HJ and Benson WL: Immature (malignant) teratoma of the ovary: A clinical and pathologic study of 58 cases. Cancer 37: 2359-2372, 1976.

5. Byrd K, Stany MP, Herbold NC, Leath CA III and Hamilton CA: Growing teratoma syndrome: Brief communication and algorithm for management. Aust NZ J Obstet Gynaecol 53: 318-321, 2013.

6. Ustunyurt E, Gungor T, Iskender C, Ustunyurt BO, Bilge U and Mollamahmutoglu L: Tumor markers in mature cystic teratomas of the ovary. Arch Gynecol Obstet 279: 145-147, 2009.

7. Bast RC Jr, Badgwell D, Lu Z, Marquez R, Rosen D, Liu J, Baggerly KA, Atkinson EN, Skates S, Zhang Z, et al: New tumor markers: CA125 and beyond. Int J Gynecol Cancer 15 (Suppl 3): 274-281, 2005.

8. Harada M, Osuga Y, Fujimoto A, Fujimoto A, Fujii T, Yano T and Kozuma S: Predictive factors for recurrence of ovarian mature cystic teratomas after surgical excision. Eur J Obstet Gynecol Reprod Biol 171: 325-328, 2013. 